A point of practical importance is that the $d$ 's (which are, in fact, correlation coefficients) do not exceed 1 in magnitude; the same is true of the $r$ 's. For this reason, the iterative scheme can easily be programmed using fixed-point arithmetic, though double-precision working is frequently needed in the calculation of the sines and cosines.

Rothamsted Experimental Station

M. J. R. HEALY

Harpenden

Herts., England

1. H. HotelLING, "Relations between two sets of variates," Biometrika, v. 28, 1936, p. 321-377;

2. A. C. ArtKen, "The evaluation, with applications, of a certain triple product matrix,' Roy. Soc. Edinburgh, Proc., v. 57, 1936, p. 172-181.

3. A. S. HouseHOLDER, Principles of Numerical Analysis (Sect. 4.115), McGraw-Hill Book Co., Inc., New York, 1953.

\title{
Polynomial Approximations to Bessel Functions of Order Zero and One and to Related Functions
}

In the course of preparing a programme for a large digital computer, the following formulas, which are of use in the calculation of Bessel functions on such machines, have been obtained. The methods of obtaining the approximations are those described by Lanczos in NBS AMS 9 [1], with trivial modifications (a complete set of references is given in [1]). The results enable $J_{0}(x), J_{1}(x), Y_{0}(x)$, $x Y_{1}(x)$, and $\bar{J}_{0}(x)$ to be calculated for all values of $x$ and $K_{0}(x), K_{1}(x)$, and $\bar{K}_{0}(x)$ to be calculated for values of $x$ exceeding unity; where we define

and

$$
\bar{J}_{0}(x)=\int_{0}^{x} J_{0}(t) d t
$$

$$
\bar{K}_{0}(x)=\int_{0}^{x} K_{0}(t) d t .
$$

We define auxiliary functions such that

$$
\begin{array}{lrl}
J_{n}(x)=\sqrt{\frac{2}{\pi x}}\left(P_{n}(x) \cos \left(x-\frac{n \pi}{2}-\frac{\pi}{4}\right)-Q_{n}(x) \sin \left(x-\frac{n \pi}{2}-\frac{\pi}{4}\right)\right), & n=0,1, \\
Y_{n}(x)=\sqrt{\frac{2}{\pi x}}\left(P_{n}(x) \sin \left(x-\frac{n \pi}{2}-\frac{\pi}{4}\right)+Q_{n}(x) \cos \left(x-\frac{n \pi}{2}-\frac{\pi}{4}\right)\right), & n=0,1, \\
\bar{y}_{n}(x)=\left(J_{n}(x) \log \frac{x}{2}-\frac{\pi}{2} Y_{n}(x)\right), & n=0,1, \\
K_{n}(x)=e^{-x} \sqrt{\frac{\pi}{2 x}} G_{n}(x), & n=0,1, \\
J_{0}(x)=1-\sqrt{\frac{\pi}{2 x}}\left(\bar{P}_{0}(x) \cos \left(x+\frac{\pi}{4}\right)-\bar{Q}_{0}(x) \sin \left(x+\frac{\pi}{4}\right)\right), & \\
\bar{K}_{0}(x)=\frac{\pi}{2}-e^{-x} \sqrt{\frac{\pi}{2 x}} \bar{G}_{0}(x) . &
\end{array}
$$


Then with the maximum error stated in brackets in each case, and provided $0<t<1$

$$
\begin{aligned}
& J_{0}(4 t)=1.00000,00000-3.99999,98721 t^{2}+3.99999,73021 t^{4} \\
& -1.77775,60599 t^{6}+0.44435,84263 t^{8}-0.07092,53492 t^{10} \\
& +0.00767,71853 t^{12}-0.00050,14415 t^{14}\left(10 \times 10^{-10}\right) \\
& \frac{1}{t} J_{1}(4 t)=1.99999,99998-3.99999,99710 t^{2}+2.66666,60544 t^{4} \\
& -0.88888,39649 t^{6}+0.17775,82922 t^{8}-0.02366,16773 t^{10} \\
& +0.00220,69155 t^{12}-0.00012,89769 t^{14}\left(3 \times 10^{-10}\right) \\
& \frac{1}{\sqrt{2 \pi}} P_{0}\left(\frac{4}{t}\right)=\underset{-0.00004,87613 t^{6}+0.00001,73565 t^{8}-0.00017,34300 t^{4}}{0.00000,37043} \\
& -0.00004,87613 t^{6}+0.00001,73565 t^{8}-0.00000,37043 t^{10} \\
& \left(12 \times 10^{-10}\right) \\
& \begin{aligned}
\frac{1}{t} \sqrt{\frac{1}{2 \pi}} Q_{0}\left(\frac{4}{t}\right)= & -0.01246,69441+0.00045,64324 t^{2}-0.00008,69791 t^{4} \\
& +0.00003,42468 t^{6}-0.00001,42078 t^{8}+0.00000,32312 t^{10}
\end{aligned} \\
& \left(25 \times 10^{-10}\right) \\
& \frac{1}{\sqrt{2 \pi}} P_{1}\left(\frac{4}{t}\right)=0.39894,22819+0.00292,18256 t^{2}-0.00022,32030 t^{4} \\
& +0.00005,80759 t^{6}-0.00002,00920 t^{8}+0.00000,42414 t^{10} \\
& \left(15 \times 10^{-10}\right) \\
& \frac{1}{t} \sqrt{\frac{1}{2 \pi}} Q_{1}\left(\frac{4}{t}\right)=0.03740,08364-0.00063,90400 t^{2}+0.00010,64741 t^{4} \\
& -0.00003,98708 t^{6}+0.00001,62200 t^{8}-0.00000,36594 t^{10} \\
& \left(25 \times 10^{-10}\right) \\
& \bar{y}_{0}(4 t)=-0.57721,56649-1.69113,74142 t^{2}+3.69113,88793 t^{4} \\
& -2.23311,02234 t^{6}+0.66943,21484 t^{8}-0.12141,87561 t^{10} \\
& +0.01489,99271 t^{12}-0.00135,08487 t^{14}+0.00008,91322 t^{16} \\
& \left(6 \times 10^{-10}\right) \\
& 4 t \bar{y}_{1}(4 t)=1.00000,00004-0.61772,53972 t^{2}-10.76454,72724 t^{4} \\
& +11.62078,91416 t^{6}-4.91052,91148 t^{8}+1.14180,33012 t^{10} \\
& -0.16910,81720 t^{12}+0.01699,21876 t^{14}-0.00102,66368 t^{16} \\
& \left(6 \times 10^{-10}\right) \\
& \begin{aligned}
\sqrt{\frac{\pi}{2}} G_{0}\left(\frac{1}{t}\right)= & 1.25331,41373-0.15666,41816 t+0.08811,12782 t^{2} \\
& -0.09139,09546 t^{3}+0.13445,96228 t^{4}-0.22998,50328 t^{5} \\
& +0.37924,09730 t^{6}-0.52472,77331 t^{7}+0.55753,68367 t^{8} \\
& -0.42626,32912 t^{9}+0.21845,18096 t^{10}-0.06680,97672 t^{11} \\
& +0.00918,93830 t^{12}\left(10 \times 10^{-10}\right)
\end{aligned} \\
& \begin{aligned}
\sqrt{\frac{\pi}{2}} G_{1}\left(\frac{1}{t}\right)= & 1.25331,41373+0.46999,27013 t-0.14685,82957 t^{2} \\
& +0.12804,26636 t^{3}-0.17364,31637 t^{4}+0.28476,18149 t^{5}
\end{aligned} \\
& -0.45943,42117 t^{6}+0.62833,80681 t^{7}-0.66322,95430 t^{8} \\
& +0.50502,38576 t^{9}-0.25813,03765 t^{10}+0.07880,00118 t^{11} \\
& -0.01082,41775 t^{12}\left(10 \times 10^{-10}\right)
\end{aligned}
$$




$$
\begin{aligned}
\frac{1}{t} \bar{J}_{0}(8 t)= & 7.99999,99990-42.66666,64204 t^{2}+102.39999,00866 t^{4} \\
& -130.03159,01993 t^{6}+101.13454,38222 t^{8}-52.95243,71745 t^{10} \\
& +19.89838,57672 t^{12}-5.60245,72363 t^{14}+1.20146,77449 t^{16} \\
& -0.18673,71001 t^{18}+0.01624,75465 t^{20}\left(10 \times 10^{-10}\right) \\
\sqrt{\frac{2}{\pi}} \bar{P}_{0}\left(\frac{8}{t}\right)= & 0.79788,45600-0.01256,42405 t^{2}+0.00178,70944 t^{4} \\
& -0.00067,40148 t^{6}+0.00041,00676 t^{8}-0.00025,43955 t^{10} \\
& +0.00011,07299 t^{12}-0.00002,26238 t^{14}\left(8 \times 10^{-10}\right) \\
\frac{1}{t} \sqrt{\frac{2}{\pi}} \bar{Q}_{0}\left(\frac{8}{t}\right)= & -0.06233,47304+0.00404,03539 t^{2}-0.00100,89872 t^{4} \\
& +0.00053,66169 t^{6}-0.00039,92825 t^{8}+0.00027,55037 t^{10} \\
& -0.00012,70039 t^{12}+0.00002,68482 t^{14}\left(8 \times 10^{-10}\right) \\
\sqrt{\frac{\pi}{2}} \bar{G}_{0}\left(\frac{1}{t}\right)= & 1.25331,39163-0.78323,44963 t+1.25733,12033 t^{2} \\
& -3.09054,43850 t^{3}+9.02560,45356 t^{4}-25.43912,19592 t^{5} \\
& +60.46288,82856 t^{6}-112.80726,52384 t^{7}+158.83274,70627 t^{8} \\
& -163.74821,02377 t^{9}+119.22659,27008 t^{10}-57.88900,96515 t^{11} \\
& +16.78876,58787 t^{12}-2.19748,24449 t^{13}\left(25 \times 10^{-10} \text { in }_{\bar{K}}\right) .
\end{aligned}
$$

An attempt to derive similar formulas for $I_{0}(x), I_{1}(x)$, and $\bar{I}_{0}(x)$ by the same methods proved unsuccessful, since these functions cannot be defined by a differential equation and a boundary condition at infinity.

\section{A. J. M. Нiтchсоск}

United Kingdom Atomic Energy Authority

Industrial Group Headquarters

Risley, Warrington,

Lancashire, England

1. NBS Applied Mathematics Series, No. 9, Tables of Chebyshev Polynomials $S_{n}(x)$ and $C_{n}(x)$, U. S. Govt. Printing Office, Washington, D. C., 1952, Introduction.

\section{An Iterative Method for the Solution of Linear Equations Based on the Power Method for Proper Vectors}

Introduction. A computing machine program for obtaining the largest proper value and proper vector of an $(n+1, n+1)$ matrix $A$ by the power method (i.e., by the iteration $X_{\nu+1}=A X_{\nu}$ ) may be used for solving a system of $n$ linear equations in $n$ unknowns. In the following note a method of setting up the iteration together with a simple criterion for convergence of the iteration are given.

Method. Let $B$ be an $(n+1, n+1)$ matrix with real elements and real proper value $k$ such that $|k|$ is greater than the moduli of all the remaining proper values of $B$. If $X$ is a non-exceptional non-zero column vector, $X=\operatorname{col}\left(\xi_{1}, \xi_{2}, \cdots, \xi_{n}, \xi_{n+1}\right)$, with real components, it is well known that the iteration $X_{0}=X, X_{1}=B X_{0}$, $\cdots, X_{\nu+1}=B X_{\nu}$ is such that $X_{\nu+1} \doteq k X_{\nu}$. (There are exceptional choices of $X$ for which the iteration will not converge to the largest proper value and vector; but round-off error during the iteration will usually make the $X_{\nu}$ non-exceptional 